\title{
Genetic insights beginning to divide autism diagnosis
}

News has already emerged that the American Psychiatric Association is considering expanding its definition of autism to include a broader range of developmental and mental syndromes in the fifth edition of the Diagnostic and Statistical Manual of Mental Disorders. But under the radar, geneticists are moving in the opposite direction, parsing the disease into more specific subtypes on the basis of its molecular etiology.

Autism is not a biological disorder, but rather a behavioral description, with the underlying disease "a constellation of rare genetic disorders," says John Spiro, the senior associate director for research at the New York-based Simons Foundation Autism Research Initiative. Last month, Spiro spoke at the first international symposium on a genetic subtype of autism known as PhelanMcDermid syndrome, an event that took place at the New York Academy of Medicine.

Stephen Scherer, director of the Centre for Applied Genomics at The Hospital for Sick Children in Toronto echoes Spiro's view, saying that autism may in fact be a "bunch of different genetic disorders that have a common clinical outcome."

For example, Rett syndrome, a neurodevelopmental disorder that affects primarily girls and falls under the broad 'autism spectrum disorder' umbrella, has a distinct genetic cause - a mutation typically in the $M E C P 2$ gene on the $\mathrm{X}$ chromosome, enabling diagnosis of affected individuals. The genetic subtype was first described in 1999 (Nat. Genet. 23, 185-188, 1999).

Researchers are just beginning to parse other subtypes on the basis of genetics and,

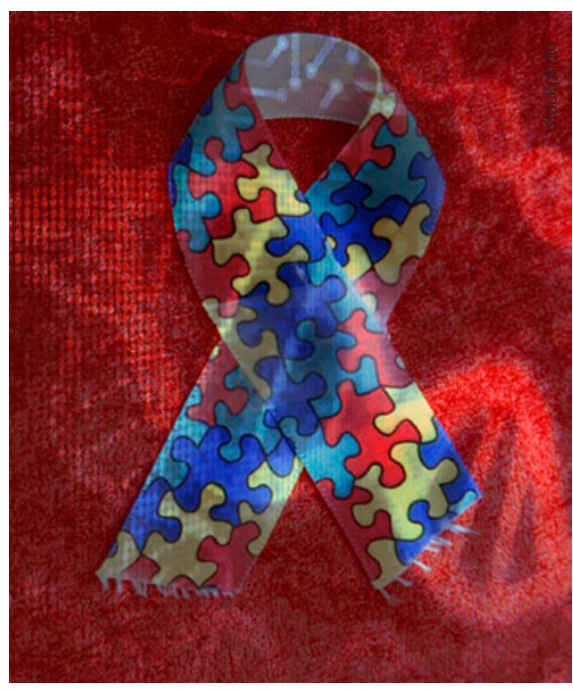

Puzzling out: Genotyping autism subtypes.

in particular, copy number variants, where entire portions of a chromosome are either deleted or duplicated. A tiny deletion on chromosome 22 is behind Phelan-McDermid syndrome and is estimated to occur in fewer than 1 in 10,000 pregnancies. Deletions and duplications on chromosomes 16, 15 and 1, meanwhile, have also been identified and are being considered as separate subtypes of autism by geneticists. These subtypes do not have lay names and are simply referred to by their chromosomal aberration. For instance, autism caused by mutations to chromosome 16 is now often being called 16p11.2 (or 16p for short) and has been estimated to occur in about $1 \%$ of all autism cases (N. Engl. J. Med. 358, 667-675, 2008).
Each of these rare genetic events contributes to only $1 \%$ to $2 \%$ of autism cases, and collectively they so far explain only $15 \%$ of total autism cases (Arch. Neurol. $67,395-399,2010)$. Yet, as the ability to study individuals' DNA at greater and greater resolution improves, scientists are uncovering more rare copy number variants and other genetic events associated with autism.

Scherer has initiated a study to sequence the protein-coding regions of DNA from around 1,000 people with autism, and he predicts that this study and others like it will eventually uncover the genetic roots of half of all autism cases. This should go a long way to helping parents and children come to grips with diagnoses of autism, says Scherer. "We're finding that families like to learn what the underlying cause of autism is."

Frequently, however, genetic diagnoses are not clinically useful because they so far haven't correlated with disease severity, prognosis or a particular treatment, notes Louise Gallagher, a child psychiatrist at Trinity College Dublin. Yet she acknowledges that the field is moving toward genetic subtyping. "We have this big category we call autism, but perhaps within that there are different syndromes," she says.

Ultimately, identifying syndromes on the basis of a person's genetic background could have huge implications for treatment by identifying the pathways and mechanisms involved, says Spiro. "Understanding the etiology completely changes the playing field."

Monica Heger

\section{France promotes research into rare diseases with new plan}

PARIS - On 28 February, international Rare Disease Day, the French government finally unveiled a four-year plan to bolster research and treatment into this group of ailments, which include sickle anemia and amyotrophic lateral sclerosis.

The plan, which spans from this year to 2014 , aims to improve the quality of care for people with rare diseases, bolster research and expand European and international cooperation in this area. It is a continuation of France's first National Plan for Rare Diseases, which ran from 2005 to 2008 and was claimed to be a world first. (A second iteration of the plan was due to be launched in 2010 , but it was delayed in part because of a government reshuffle in November.)

With a $€ 180$ million ( $\$ 250$ million) budget, the plan aims to keep France as a European leader for research in rare diseases, the country's Research Minister Valérie Pécresse said at a press conference that coincided with the release of the new plan. Figures from Orphanet, the European portal of information on orphan drugs, show that France's 122 rare disease registries puts it at the top in Europe; Germany, by comparison, has 86 .

Of the $€ 180$ million total budget, the
National Research Agency will allocate at least $€ 40$ million over the next four years. And, according to the new French plan, a forthcoming foundation will coordinate work on rare diseases, whereas a new national database will track epidemiological details about the incidence of rare diseases with support from Orphanet. Genzyme France, the Paris-based French subsidiary of the Cambridge, Massachusetts-based biotech, which claims to be a pioneer in rare disease drugs, welcomes the plan. "It represents a number of major advances, especially for research," says René Thomas, director of Genzyme France's therapeutic division. The 